\title{
Assimilation and High Resolution Forecasts of Surface and Near Surface Conditions for the 2010 Vancouver Winter Olympic and Paralympic Games
}

\author{
Natacha B. Bernier, ${ }^{1}$ StéPhane Bélair, ${ }^{2}$ Bernard Bilodeau, ${ }^{2}$ and Linying Tong ${ }^{3}$
}

\begin{abstract}
A dynamical model was experimentally implemented to provide high resolution forecasts at points of interests in the 2010 Vancouver Olympics and Paralympics Region. In a first experiment, GEM-Surf, the near surface and land surface modeling system, is driven by operational atmospheric forecasts and used to refine the surface forecasts according to local surface conditions such as elevation and vegetation type. In this simple form, temperature and snow depth forecasts are improved mainly as a result of the better representation of real elevation. In a second experiment, screen level observations and operational atmospheric forecasts are blended to drive a continuous cycle of near surface and land surface hindcasts. Hindcasts of the previous day conditions are then regarded as today's optimized initial conditions. Hence, in this experiment, given observations are available, observation driven hindcasts continuously ensure that daily forecasts are issued from improved initial conditions. GEM-Surf forecasts obtained from improved short-range hindcasts produced using these better conditions result in improved snow depth forecasts. In a third experiment, assimilation of snow depth data is applied to further optimize GEM-Surf's initial conditions, in addition to the use of blended observations and forecasts for forcing. Results show that snow depth and summer temperature forecasts are further improved by the addition of snow depth data assimilation.
\end{abstract} system.

Key words: Point forecast, assimilation, land surface forecast

\section{Introduction}

The 2010 Winter Olympic and Paralympic Games were held in and around Vancouver, British Columbia. Competition sites were diverse and distributed across urban and alpine areas. In the alpine region, orography

1 Meteorological Research Division, Environment Canada, Dorval, QC H9P 1J3, Canada. E-mail: natacha.bernier@ec.gc.ca

2 Meteorological Research Division, Environment Canada, Dorval, QC, Canada.

3 Canadian Meteorological Center, Environment Canada, Dorval, QC, Canada. is complex with substantial variations on scales much smaller than several kilometers. Elevation mapped on a 100-m grid spacing (Fig. 1) clearly demonstrates that traveling a short distance can result in a large elevation difference. In the alpine area, distance traveled during an Olympic event such as skiing from the top to the bottom of the ski downhill competition courses is enough to experience substantial changes in weather conditions. For example, the temperature difference across the $853 \mathrm{~m}$ drop of the Dave Murray Downhill course can exceed $5^{\circ}$. A skier traveling down such slope can also experience variable weather on the way to the bottom (e.g., rain, snow, fog) that cannot be resolved by the regional and global deterministic prediction systems whose grid spacing are such that the entire downhill course and much of the surrounding area is represented by a single grid point.

The spatially variable weather conditions that result from such complex local orography are typically not well represented by large scale operational forecast systems. In Canada, the two operational forecast models from which official products were issued for the Vancouver region are the Global Environmental Multiscale (GEM) $15 \mathrm{~km}$ short-range regional deterministic prediction system (GEM-15km, MAILHot et al. 2006) and the $33 \mathrm{~km}$ medium-range global deterministic prediction system (GEM-33km, BÉLAIR et al. 2009). When and where long observation records were available prior to VO2010, statistical packages were available to correct forecasts based on a history of forecast errors. Such statistical corrections can however require a substantial amount of data. For example, the updateable model output statistics (UMOS) used to refine Environment Canada's operational forecasts is known to require about 2 years of observations in order to accurately assess error corrections (WiLSON and VALLÉE, 2003). In remote areas, 

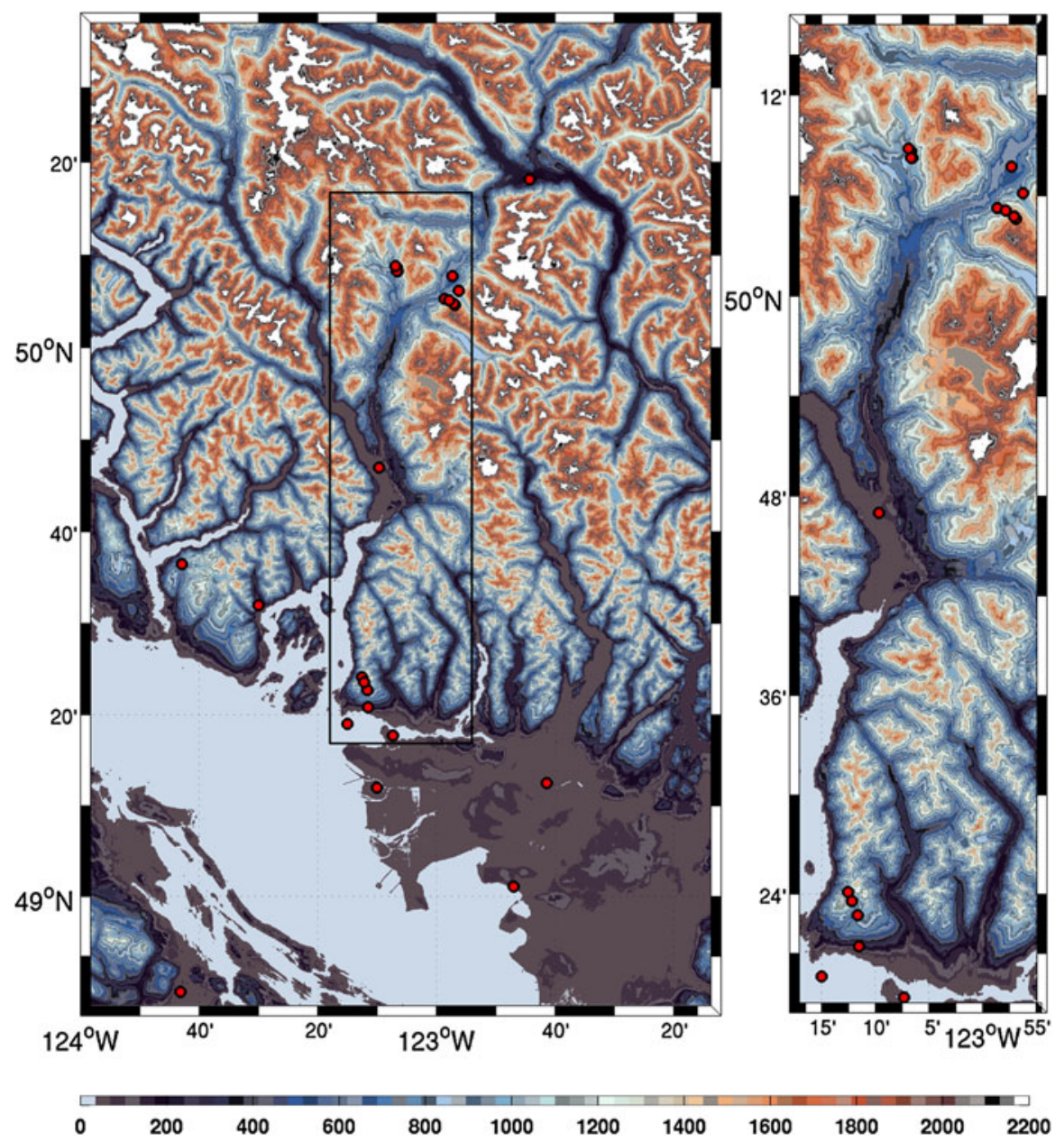

Figure 1

Observation stations and co-located GEM-Surf grid points (left see Table 1). The black box shows the region plotted in the right panel. The background colors represent orography $(\mathrm{m})$ based on the $20 \mathrm{~m}$ Canadian Digital Elevation Data (CDED50) aggregated to $100 \mathrm{~m}$ grid spacing

there is often insufficient data to correct forecasts for all points of interest.

Special events such as the Olympic Games can bring much attention to a remote region where long established observation networks may not be well developed and are unlikely to be deployed early enough to optimize the training of like UMOS systems. This was the case for the VO2010 region where few observation stations were present before the deployment of the SNOW-V10 observation network in preparation for the Games (e.g., IsAAC et al. 2012; JoE et al. 2012; GultePE et al. 2012). High resolution forecasts were thus needed to provide officials and athletes with weather information refined to the local scale using systems that require little to no data training.
A suite of experimental high resolution forecast systems and products specifically tailored for the VO2010 (MaILHot et al. 2010) were developed and implemented to complement the relatively coarse operational GEM-15km and GEM-33km forecasts. High resolution models developed for the Games included limited area models (LAMs) with 2.5 and $1 \mathrm{~km}$ grid spacing (MAILHOT et al. 2012) and a $100 \mathrm{~m}$ grid spacing near surface and land surface forecast model (BERNIER et al. 2011). The goal of this present study was to complement the gridded systems by adding a simple but robust system that could be quickly modified to include new stations as they came online, with no limitation to resolution and spatial distribution. 
The addition is in the form of a near surface and land surface point forecast system, improved by a more realistic description of land surface conditions (e.g., elevation and land cover type) and better land surface initial conditions (e.g., surface temperature and snow depth). This new system was driven using operational atmospheric forecasts instead of the experimental LAM high resolution forecast systems since they are updated several times daily and are available nation wide, a requirement for any future extension of the land surface system. In this testbed version, 26 sites scattered throughout the Olympics region and collocated with observation stations were selected for development and implementation (Fig. 1; Table 1). The sites include low lying stations, as well as alpine stations.

The outline of this paper is as follows. The near surface and land surface forecast system is briefly defined in Sect. 2 along with the assimilation of temperature and snow depths. The three experiments used to evaluate the impact of assimilating observed values are also described in this section. Forecast results for temperature, dew point temperature, and snow depth are presented and evaluated in Sect. 3. The last section is a discussion of the work presented in this article and a brief description of how such a system could be expanded to span the country and included as an operational product.

\section{Near Surface and Land Surface Forecast System}

In this study, a middle range complexity land surface model based on the interactions between soil, biosphere, and atmosphere, ISBA (NoILHAN and Planton, 1989; Douville et al. 1995) is used together

Table 1

World Meteorological Organization (WMO) observation stations

\begin{tabular}{|c|c|c|c|c|c|}
\hline WMO ID ${ }^{b}$ & Station code & Station name & Longitude $\left({ }^{\circ} \mathrm{W}\right)$ & Latitude $\left({ }^{\circ} \mathrm{N}\right)$ & Elevation $(\mathrm{m})$ \\
\hline 1108987 & $\mathrm{VOA}^{\mathrm{a}}$ & Whistler Mt. High Level & 122.9476 & 50.0770 & $1,639.97$ \\
\hline 1108988 & $\mathrm{VOB}^{\mathrm{a}}$ & Whistler Mt. Low Level & 122.9770 & 50.0878 & 933.13 \\
\hline 1100875 & VOC $^{\mathrm{a}}$ & Blackcomb Mt. Base & 122.9546 & 50.1290 & 659.00 \\
\hline 1101300 & VOD $^{a}$ & Callaghan Valley & 123.1106 & 50.1442 & 869.00 \\
\hline 1102255 & $\operatorname{VOE}^{\mathrm{a}}$ & Cypress Bowl North & 123.2093 & 49.4019 & 953.00 \\
\hline 1102256 & VOG & Cypress Bowl South & 123.1937 & 49.3786 & 960.00 \\
\hline 1108989 & $\mathrm{VOH}$ & Whistler Mt. High Level & 122.9513 & 50.0793 & $1,643.00$ \\
\hline 1100881 & $\mathrm{VOI}^{\mathrm{a}}$ & Blackcomb Base Sliding Center Top & 122.9375 & 50.1024 & 937.00 \\
\hline 1108990 & VOL & Whistler Mt. Mid-Station & 122.9643 & 50.0853 & $1,320.00$ \\
\hline 1046332 & $\mathrm{VOM}^{\mathrm{a}}$ & Port Mellon & 123.5000 & 49.5333 & 122.57 \\
\hline 1015630 & $\mathrm{VOO}^{\mathrm{a}}$ & North Cowichan & 123.7186 & 48.8242 & 60.00 \\
\hline 1046392 & $\mathrm{VOP}^{\mathrm{a}}$ & Powell River & 124.4993 & 49.8341 & 125.00 \\
\hline 1026562 & $\operatorname{VOQ}^{\mathrm{a}}$ & Qualicum Airport & 124.4000 & 49.3333 & 58.20 \\
\hline 1047172 & $\mathrm{VOU}^{\mathrm{a}}$ & Sechelt & 123.7142 & 49.6080 & 86.00 \\
\hline 1100001 & VOW & Callaghan Valley Ski Jump Top & 123.1089 & 50.1377 & 936.00 \\
\hline 1101320 & VOX & Callaghan Valley Ski Jump Bottom & 123.1106 & 50.1377 & 857.00 \\
\hline 1100003 & VOY & Callaghan Valley Biathlon & 123.1147 & 50.1469 & 857.00 \\
\hline 1100004 & VOZ & Cypress Bowl Event Freestyle & 123.2026 & 49.3929 & 958.00 \\
\hline 1086082 & $\mathrm{WGP}^{\mathrm{a}}$ & Pemberton Airport & 122.7378 & 50.3025 & 204.30 \\
\hline 1108446 & WHC & Vancouver Harbour & 123.1219 & 49.2954 & 2.50 \\
\hline 1106178 & WMM & Pitt Meadows & 122.6902 & 49.2083 & 5.00 \\
\hline 1106200 & WSB & Point Atkinson & 123.2500 & 49.3167 & 35.00 \\
\hline $10476 \mathrm{~F} 0$ & $\mathrm{WSK}^{\mathrm{a}}$ & Squamish Airport & 123.1611 & 49.7833 & 52.10 \\
\hline 1108824 & $\mathrm{WWA}^{\mathrm{a}}$ & West Vancouver & 123.1919 & 49.3472 & 168.00 \\
\hline 1108910 & WWK & White Rock & 122.7828 & 49.0183 & 13.00 \\
\hline 1108447 & YVR & Vancouver International Airport & 123.1667 & 49.2000 & 4.30 \\
\hline
\end{tabular}

${ }^{a}$ Indicate that station is equipped with a snow depth sensor

b Station identifier 
with downscaling of atmospheric forcing and detailed land surface conditions to produce near surface and land surface forecasts adapted to local conditions. In such an approach, there are no horizontal interactions between the model grid points (e.g., due to advection and diffusion). The near surface and land surface (GEM-Surf) forecast system includes a prognostic evolution of snow properties such as skin and deep snow temperature, snow surface albedo, snow mass, mean snow density and liquid water contained in the snow canopy (BÉLAIR et al. 2003a). Yet, the cost of the integration is small enough for the system to be run several times daily at grid spacings of some one or two hundred meters across the populated areas of a country as large as Canada.

In this study, 26 grid points were centered over observation stations located within the VO2010 region (Fig. 1, red dots; Table 1). Since there are no horizontal interactions between adjacent cells, the computational grid can be made of a gathering of unevenly distributed and unevenly sized grid points. One novel aspect of such a forecast system is, thus, that the computational grid can be made of an array of varying grid spacings with no limitation to the change in resolution between adjacent cells. For VO2010, all grid spacings were set to $100 \mathrm{~m}$. Reported station elevations were used for model elevation while vegetation covering the $100 \mathrm{~m}$ surrounding each station/ grid point was estimated from high resolution aerial photographs and camera displays when available. Typical vegetation classes for a ski slope area were found to be $80 \%$ evergreen needle-leaf trees (representing the coniferous forests) and $20 \%$ short grass and forbs representing the ski slope. All other characteristics such as soil type, vegetation class, and leaf area index (for stations where images are not available) were extracted from databases such as the 20-m Canadian Digital Elevation Data, the Earth Observation for Sustainable Development of the forest, GlobCover, and Agriculture and Agri-Food Canada geophysical databases. All data used for this study is publicly available through http://www.geobase.ca/ geobase/en/index.html.

The first analysis of surface fields such as soil moisture, snow depth, and ground temperature for each of the GEM-Surf grid points are simply interpolated from GEM- $15 \mathrm{~km}$. This step is performed only once to cold-start GEM-Surf. Fields such as snow and soil conditions are then prognostically evolved. Retaining memory of previous runs (i.e., allowing for continuous build up or accumulation) is ensured by using the previous day GEM-Surf forecast as the analysis (i.e., the starting point) for today's forecast. The model is thus restarted daily using the previous day 24th forecast hour as today's analysis. The continuous cycle of short-range integrations is used to update, daily, the land surface analyses used to initialize $96 \mathrm{~h}$ forecasts. In this study, three analysis cycles are used, they are described in Sects. 2.3 and 2.4.

The GEM-Surf forecasts are initialized with the analyses and driven by hourly downscaled operational GEM-15km atmospheric forecasts for forecast hours 1-48 and GEM-33km forecast for hours 49-96. The first model level (located at roughly $40 \mathrm{~m}$ above ground) is used for winds, temperature, and humidity. Other required variables like pressure, downwelling radiation, and precipitation are taken at the surface (Fig. 2a).

The downscaling procedures are embedded in the GEM-Surf system. The bulk of the downscaling applied to the large scale operational atmospheric forecasts is a temperature correction based on the difference between the large scale model elevation and the elevation at the point being refined. For the Olympic region, a lapse rate of $6{ }^{\circ} \mathrm{C} / \mathrm{km}$ was found to best fit the local observed conditions for all seasons and for night and day conditions. It is used to correct large scale forecasts. Surface pressure is adapted following $P_{\mathrm{hr}}=\exp \left(\left(\delta_{z} g / R\right) / T_{\mathrm{hr}}\right) P_{\mathrm{lr}}$ where $P_{\mathrm{hr}}$ is the adapted high resolution pressure, $P_{\mathrm{lr}}$ is the pressure from the low resolution models cubically interpolated to the high resolution grid, $g$ is the gravitational constant, and $R=287.05 \mathrm{~J} \mathrm{~kg}^{-1} \mathrm{~K}^{-1}$ is the gas constant for dry air. Relative humidity is interpolated to each high resolution grid point and subsequently converted to specific humidity using the newly corrected temperature and pressure fields. Other variables are indirectly adapted as a function of other environmental conditions such as the rain precipitation phase that changes to snow if the downscaled temperature falls below the freezing point (see BERNIER et al. 2011 for details). 


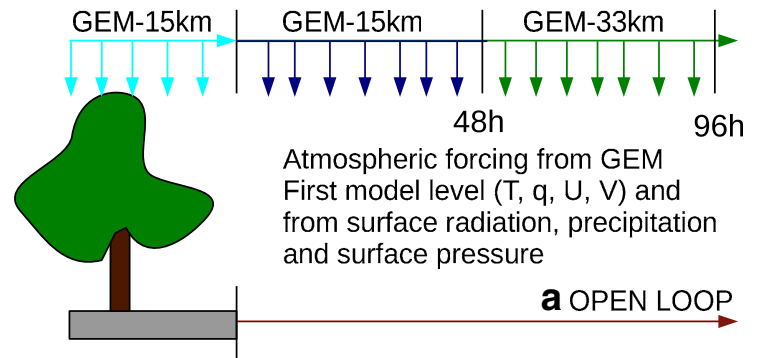

Same as open loop but observed

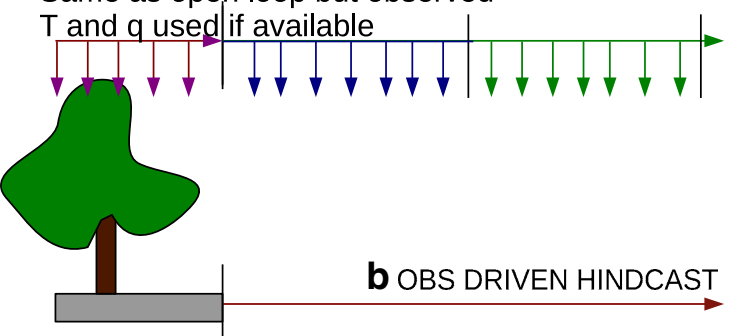

Same as obs

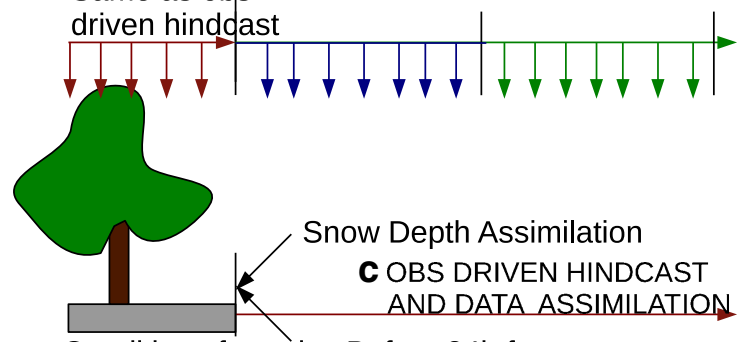

Conditions from day Before $24 \mathrm{~h}$ forecast

Figure 2

Schematics describing the point forecast application of GEM-Surf. The open loop (top), observation driven hindcast (middle) and observation driven with snow depth assimilation optimized initial conditions (bottom) are used to produce $96 \mathrm{~h}$ forecast of local conditions

\subsection{Spin-up and Forecast Period}

In this experiment, the surface fields (i.e., snow and soil conditions) were evolved at high resolution from 1 September 2007 to 31 October 2007 to allow adjustment to local conditions. This ensures, for example, that if a low lying grid point has been used to initialize a high elevation station, the freeze/thaw state will reflect the change in elevation.

Following the spin-up (under the three conditions described below), daily $96 \mathrm{~h}$ forecasts for the period 1 November 2007 to 1 April 2009 were produced using the three types of continuous analysis cycles to update the daily initial conditions as described in the sections below.

\subsection{Open Loop}

In the most straightforward application, the shortrange forecast part of the continuous (analysis) cycle is simply issued using the 24th hour of the previous forecast as initial conditions. Hence, after the initial spin-up (Sect. 2.1), the model was driven by GEM$15 \mathrm{~km}$ and GEM-33km to produce $96 \mathrm{~h}$ forecasts adjusted and refined based on local characteristics. Each new daily forecast was restarted using previous day 24th hour forecasts (Fig. 2a). It is important to stress that no observations and no data assimilation are used to drive the open loop which is thus free to evolve based on atmospheric forecast forcings only.

\subsection{Optimized Initialization}

Air temperature and humidity can rapidly modify the evolution of surface conditions especially for snow. For example, the onset of the melting season is closely related to air temperature and so in the operational snow depth analyses melting is forced based on observed temperatures (BRASNETT, 1999). Unfortunately, these analyses are too coarse to resolve local variations in snow conditions and often result in large forecast errors locally. In GEM-Surf, snow thus evolves freely from the analyses. This free evolution of the snow pack also has limits. For example, any bias in forecast temperatures will result in errors in snow depth. Since snow depth is a cumulative field, errors that result from a systematic bias can continue to accumulate over an entire season. To minimize this effect, an optimized initialization is tested.

In the second experiment (Fig. 2b), the spin-up period is modified to allow for observed temperature and humidity to replace GEM-15km and GEM- $33 \mathrm{~km}$ forecast temperature and humidity. Treatment of other atmospheric fields remained unchanged. It should be mentioned that the temperature observations were slightly perturbed using randomly sampled values between -0.5 and $0.5{ }^{\circ} \mathrm{C}$ to represent observation error.

\subsection{Data Assimilation and Optimized Initialization}

The snow modeling scheme in GEM-Surf is relatively simple (BÉLAIR et al. 2003a, b). For example, 
densification is not as sophisticated as in more complex snow models such as CROCUS and SNTHERM (BRUN et al. 1989 and JORDAN, 1991, respectively, and see SLATER et al. 2001 for a review of such models). Comparison against surface observations over the Olympic region indicate that the ISBA snow model tends to underforecast rapid melting events. Since the evolution of the snow pack is a cumulative problem, and GEM-Surf evolves freely, forecast errors both persist and accumulate over the cold season. A simple data assimilation scheme was designed to improve on GEM-Surf snow depth forecast.

In the third experiment (Fig. 2c), snow depth assimilation is thus added to the optimized initialization described in Sect. 2.3. A simple optimal interpolation technique is used to update the snow depth during the continuous cycle. Essentially, GEMSurf's previous day forecast for snow depth is used as the first guess $\left(\mathrm{Sd}^{\mathrm{f}}\right)$ which is corrected with observed snow depth $\left(\mathrm{Sd}^{\mathrm{o}}\right)$ to produce an updated snow depth. The updated snow depth $\left(\mathrm{Sd}^{\mathrm{u}}\right)$ is obtained by taking the weighted sum of the first guess and observed snow depths,

$$
\mathrm{Sd}^{\mathrm{u}}=\alpha_{\mathrm{O}} \mathrm{Sd}^{\mathrm{O}}+\alpha_{\mathrm{f}} \mathrm{Sd}^{\mathrm{f}}
$$

where $\alpha_{0}$ denotes the weight applied to the observations, and $\alpha_{\mathrm{f}}$ denotes the weight applied to the first guess. The weight of the observation is based on the variance of the first guess and the total variance such that $\alpha_{\mathrm{o}}=\sigma_{\mathrm{f}}^{2} /\left(\sigma_{\mathrm{o}}^{2}+\sigma_{\mathrm{f}}^{2}\right)$. It follows that $\alpha_{\mathrm{f}}=1-\alpha_{\mathrm{o}}$. The variance of the observations, $\sigma_{\mathrm{o}}^{2}$, is calculated using the last $6 \mathrm{~h}$ of observations and accounts for the variance of the noise which was estimated to be $2.25 \mathrm{~cm}^{2}$ following the inspection of all available observation records. The variance of the first guess, $\sigma_{\mathrm{f}}^{2}$, is given by the squared maximum of $0.1 \mathrm{Sd}^{\mathrm{f}}$ and $5 \mathrm{~cm}$.

\section{Results}

In this section, the GEM-Surf forecasts are evaluated against observations of screen level temperature, dew point temperature, and snow depth recorded at the 26 colocated grid points and observation stations (Fig. 1; Table 1). The GEM-15km and GEM-33km forecasts are also evaluated, based on interpolation to the 26 points of interest. Throughout this section, black is used to show observations, cyan is for the open loop GEM-Surf forecasts (Sect. 2.2), magenta is for GEMSurf with optimized continuous cycle (Sect. 2.3), and red is for forecasts that also include snow depth data assimilation (Sect. 2.4). Operational GEM-15km forecasts are all shown in blue while operational GEM$33 \mathrm{~km}$ forecasts are shown in green.

At first, results for screen level temperature and dew point temperature are discussed for the last GEM-Surf experiment only. GEM-Surf screen level temperature and dew point temperature forecasts from the open loop, and the two optimized initial conditions differ little except for the summer month of the alpine season. In winter, the snow temperatures in the three experiments are rapidly influenced by the atmospheric temperatures used to drive GEM-Surf. As the same downscaled temperatures are used to drive all three experiments, the screen level temperatures that result tend to resemble one another rapidly. In summer, the more complex system slightly improves on the other two systems (not shown). The more accurate representation of snow melt is associated with this improvement and will be discussed in Sect. 3.3. Given the otherwise similar forecast skill, GEM-Surf results from the combined optimized continuous cycles (i.e., improved initialization) and snow depth assimilation are presented in the temperature and dew point temperature result evaluation sections (Sects. 3.1 and 3.2, respectively).

\subsection{Screen Level Temperature}

Time series of observed and forecast screen level temperatures for 12 February to 12 March 2009 and 12 July to 9 August 2008 are shown in Fig. 3 for Blackcomb Mt. Base. The first $48 \mathrm{~h}$ of the forecasts are shown for GEM-Surf and GEM-15km (Fig. 3a, b, e, and f). Forecast hours 49-96 of GEM-Surf and GEM-33km are shown in the bottom four panels (Fig. 3c, d, g, and h).

In winter (Fig. 3a-d), forecast operational temperatures (blue and green lines) have a tendency of being too cold. In summer (Fig. 3e-h), GEM-Surf temperature forecasts (red) also improve on the GEM-15km (blue) and GEM-33km forecasts (green). It was recently demonstrated (BERNIER and BéLAIR, 

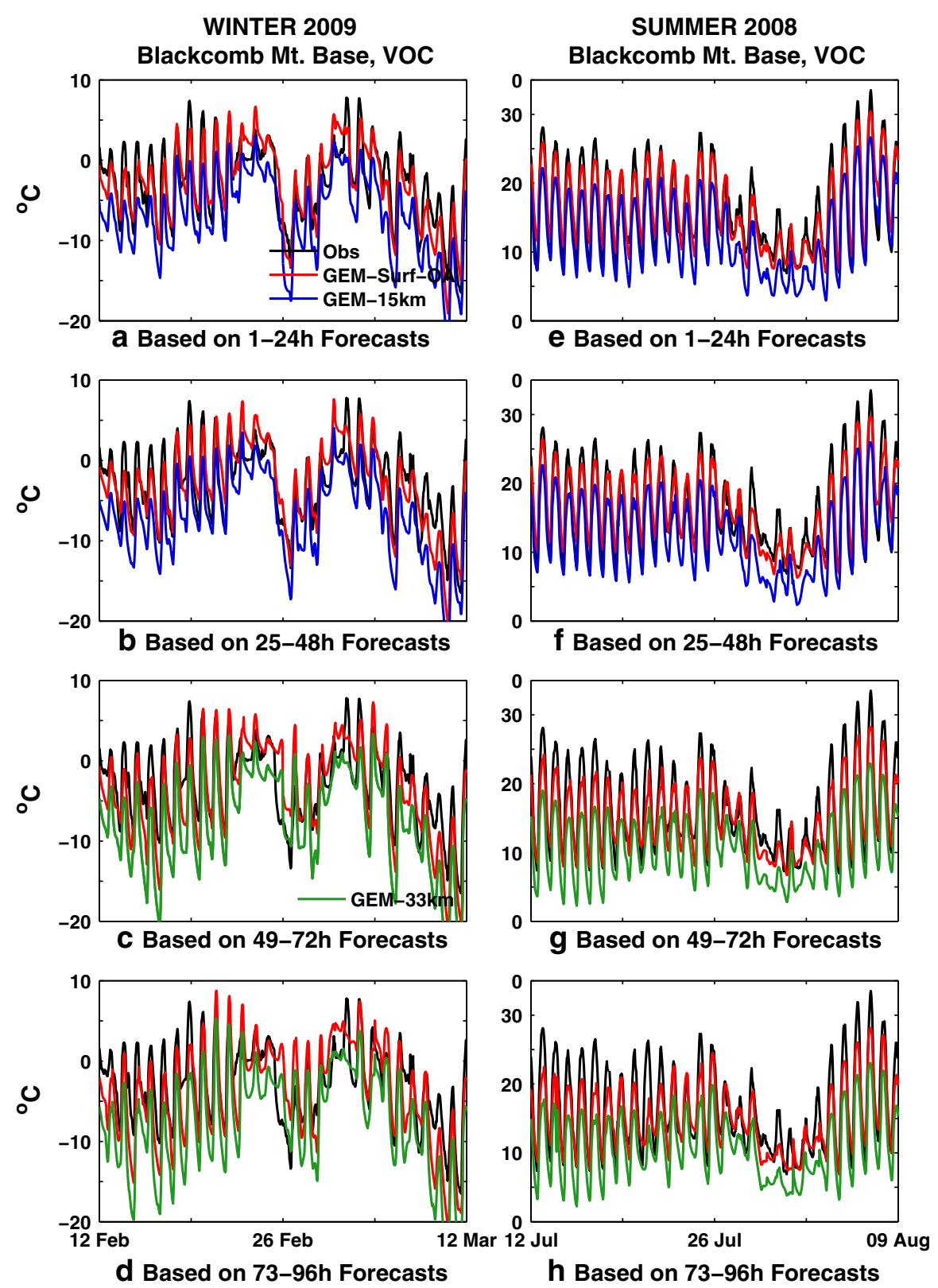

Figure 3

Observed and forecast temperatures at Blackcomb base, VOC for 12 Feb to 12 Mar 2009 (left) and 12 Jul to 09 Aug 2008 (right). Black line are observed temperatures $\left({ }^{\circ} \mathrm{C}\right)$, red line is GEM-Surf forecasts with optimized initial conditions and data assimilation forecast temperatures, blue line is GEM-15km forecasts, and green line is GEM-33km forecasts

2012) that the improvements are linked to changes in soil moisture fields that result from the more accurate modeling of the local snow conditions (i.e., cover, depth, liquid water content). In turn, the changed moisture field affects temperatures as discussed below.
Forecast errors (based on observed minus forecast temperatures) were also calculated for four seasons defined as follows: Winter, 1 January to 31 March 2008; Spring, 1 April to 30 June 2008; Summer, 1 July to 30 September 2008; and Fall, 1 October to 31 December 2008. Mean bias (solid lines) and mean 
standard deviation of the errors (STDE, dashed lines) are shown for each season on Fig. 4.

The mean bias of GEM-Surf temperature forecast error is improved for all seasons (red lines, Fig. 4a, b, e, f). The bulk of the improvement is due to the temperature correction associated with the elevation difference between the large scale GEM-15km and GEM-33km and the station elevation used in GEMSurf. The mean bias of GEM-Surf is increased for forecast hours 49-96. At day 3 and 4, GEM-33km is used to drive GEM-Surf. The slightly larger temperature variation with underlying elevation in GEM$33 \mathrm{~km}$ over GEM-15km, and the imposed lapse rate of $6{ }^{\circ} \mathrm{C} / \mathrm{km}$, explain the bulk of the increased mean bias at day 3 and 4 of the forecast.

The mean STDE of GEM-Surf is also improved for all seasons (red dashed lines, Fig. 4c, d, g, h). Reducing the variance of the error is more complicated than improving the mean bias especially since GEM-Surf does not benefit from mechanisms that prevent the overforecasting of temperature minima. The improved STDEs are primarily brought about by better land surface initial conditions and by the improved description of the surface characteristics such as vegetation, soil type, and leaf area index.

There are several factors that contribute to the cold night time temperature bias issue including the weak winds that result from orographic blocking, a numerically imposed reduction in flow to mimic flow over the rough mountains terrain that is not resolved by the GEM-15km and GEM-33km operational models (ZADRA et al. 2003). Orographic blocking was implemented to improve upper-air fields and forecasts over the Prairies, downstream of the mountains. Unfortunately, the weak winds that result in the VO2010 region lead to a decoupling of the surface and the low atmosphere. Under stable conditions, it results in plunging overnight temperatures. A maximum lapse rate $\left(8^{\circ} \mathrm{C}\right.$ between the diagnostic screen level temperature and first model layer) was thus numerically imposed to limit overnight cooling in the operational models forecast temperatures that were evaluated in this section. In GEM-Surf, there are no such numerically imposed limits. The improvement in the forecast overnight minima results from the refinement process. It shows that GEM-Surf is more than an elevation correction model which could only offset temperatures at all forecast hours in the same manner (given the fixed lapse rate used for the elevation correction). It also improves fluxes thus allowing for further improvement in forecast skill.

These positive results are encouraging and suggest that surface fluxes from the high resolution surface model could be provided to a lower resolution atmospheric model. Tests are currently underway to evaluate the impact of improving the representation of fluxes using higher resolution information.

\subsection{Screen Level Dew Point Temperature}

As for temperatures, the dew point forecast temperature differences between GEM-Surf runs are small and so only the third experiment is discussed here. The mean error statistics for dew point temperature forecast errors are shown in Fig. 5. GEM-Surf mean bias (red solid line) improves over GEM-15km (blue) and GEM-33km (green) for all seasons (Fig. 5a, b, e, f). Again, the change in forcing fields from GEM-15km to GEM-33km at forecast hour 49 is evident from the increased diurnal signal in the mean bias for days 3-4. In summer (Fig. 5e), the mean bias is also shifted by some $2{ }^{\circ} \mathrm{C}$ between day 2 and day 3. Since both operational models are based on similar assimilation systems, this large difference over the summer months is thought to be linked with the snow in GEM-15km and GEM-33km. It could also be related to the different downscaling corrections that are applied to the atmospheric forcing from the two systems. This will be discussed again in the next section. It is also interesting to note that the amplitude of the diurnal variation of the forecast is significantly reduced in GEM-Surf indicating that the dew point temperature is a better forecast throughout the $96 \mathrm{~h}$ period. Although not explicitly evaluated here, it is expected given the increase forecast skill for dew point temperature that relative humidity forecasts are also improved.

The mean STDE of dew point temperature forecasts also shows that GEM-Surf improves over the operational GEM-15km and GEM-33km except for the fall day 3 and day 4 forecasts when GEM-Surf and GEM-33km STDEs are comparable (Fig. 5c, d, g, h). The overall improvement of the dew point forecast suggests for example a potential for improved forecast 

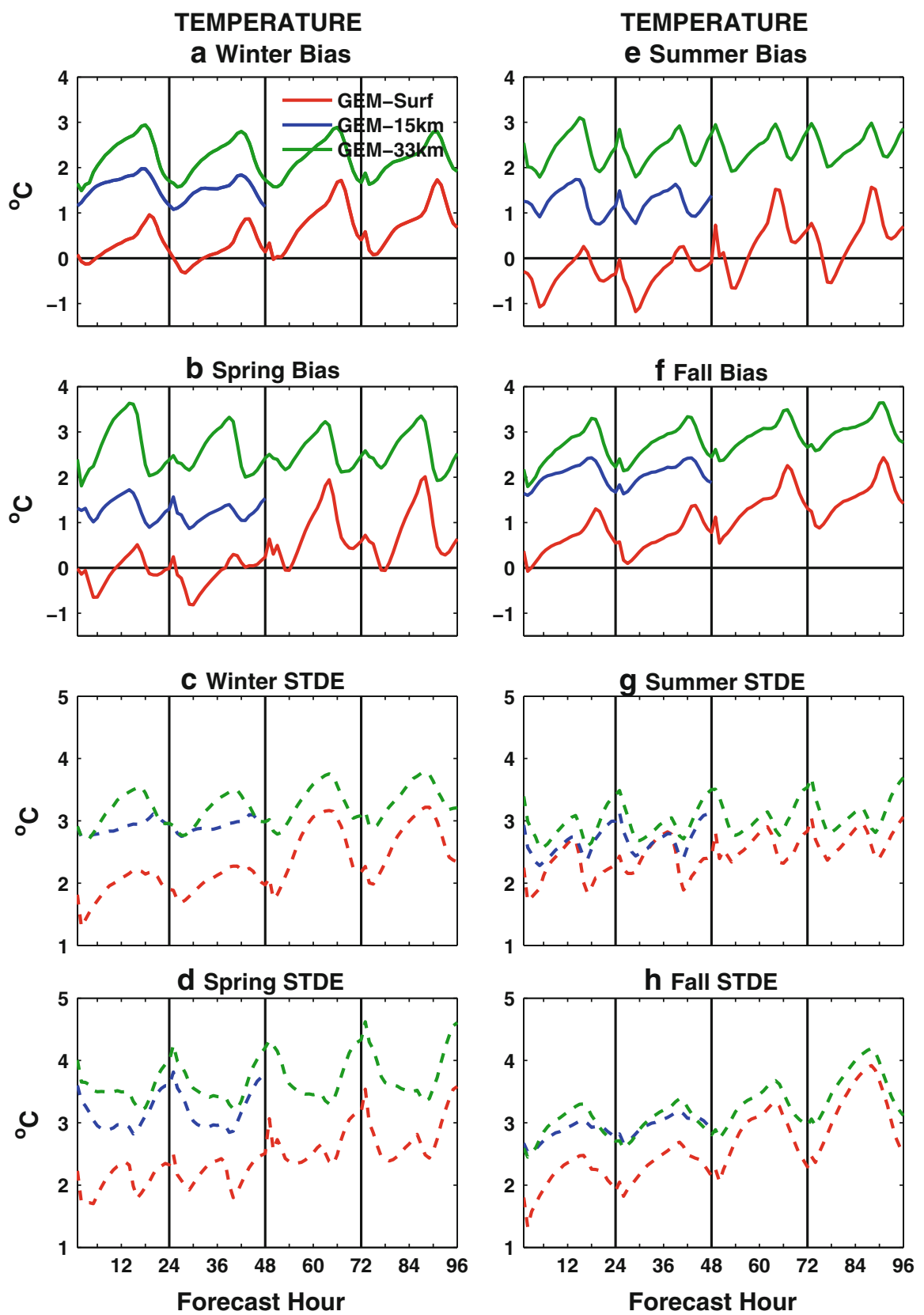

Figure 4

Seasonal temperature forecast mean bias (solid line panels $\mathbf{a}-\mathbf{d}$ and mean STDE (dashed line panels $\mathbf{e}-\mathbf{h}$ ). Scores are based on observed minus forecast values at all 26 stations. Red lines are for the optimized initial conditions and data assimilation GEM-Surf error statistics. Blue lines are for GEM-15km forecasts (forecast range is $48 \mathrm{~h}$ ). Green lines are for GEM-33km forecasts

skill of frost, fog, and is also indicative of the improved minimum temperature forecasts discussed in the previous section. It could also lead to better humidity in the atmospheric boundary layer which is an important factor for condensation, clouds, and precipitation.

\subsection{Snow Depth}

Interannual variability can be large in alpine regions. Around the VO2010 Competition Sites (e.g., Blackcomb Mt. Base), 2008 snow packs were thicker than those observed in 2009 (Fig. 6a, b, e, black lines; 


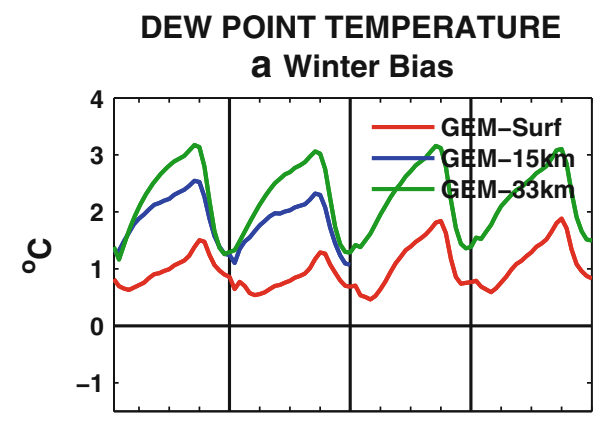

b Spring Bias
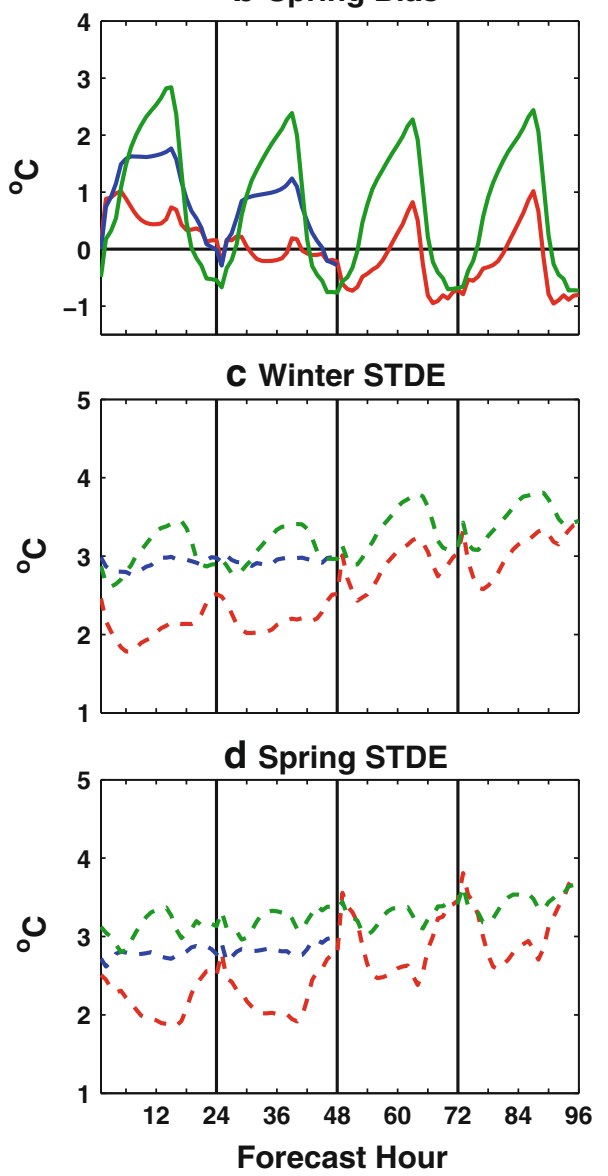

DEW POINT TEMPERATURE e Summer Bias

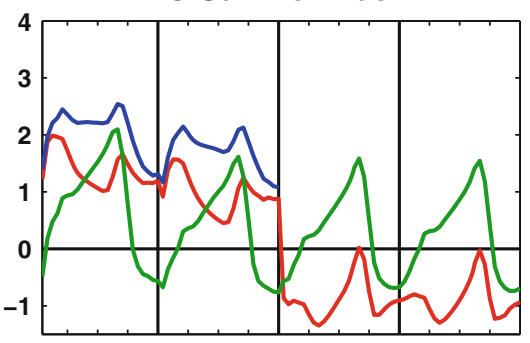

f Fall Bias

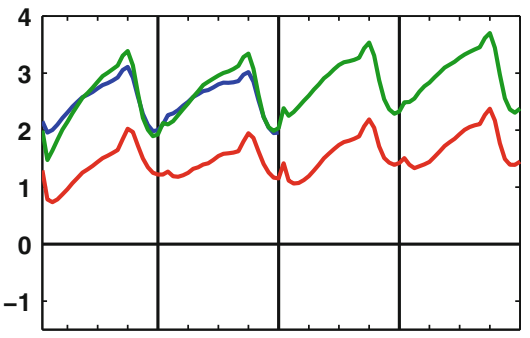

g Summer STDE
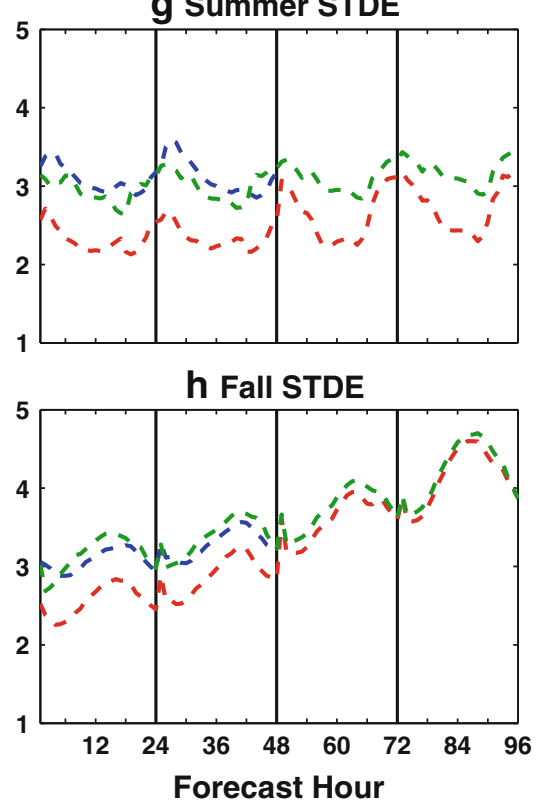

Figure 5

Same as Fig. 4 but for dew point temperature

note that the red lines often lie on top of the black lines hiding them). At low lying stations such as Squamish Airport and West Vancouver, winter temperatures are milder and snow typically melts rapidly following storm events or short accumulation periods (Fig. 6d, f, $\mathrm{g}$, and $\mathrm{h}$, black lines).

At most coastal stations (e.g., Fig. 6d, h) both GEM-15km and GEM-33km result in good forecast of the snow conditions. Over the alpine region and along valleys, both GEM-15km and GEM-33km have a tendency to overforecast the snow pack except for Cypress Bowl (Fig. 6b) where the operational model elevations are far below the real station elevation. In general, the GEM-33km overforecast is much larger than GEM-15km and the snow pack remains later in the season often persisting until July (Fig. 6a-c, e-g). 
a Blackcomb Mt. Base, VOC

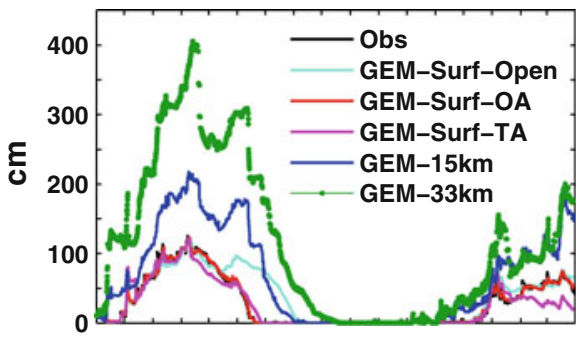

b Cypress Bowl North, VOE
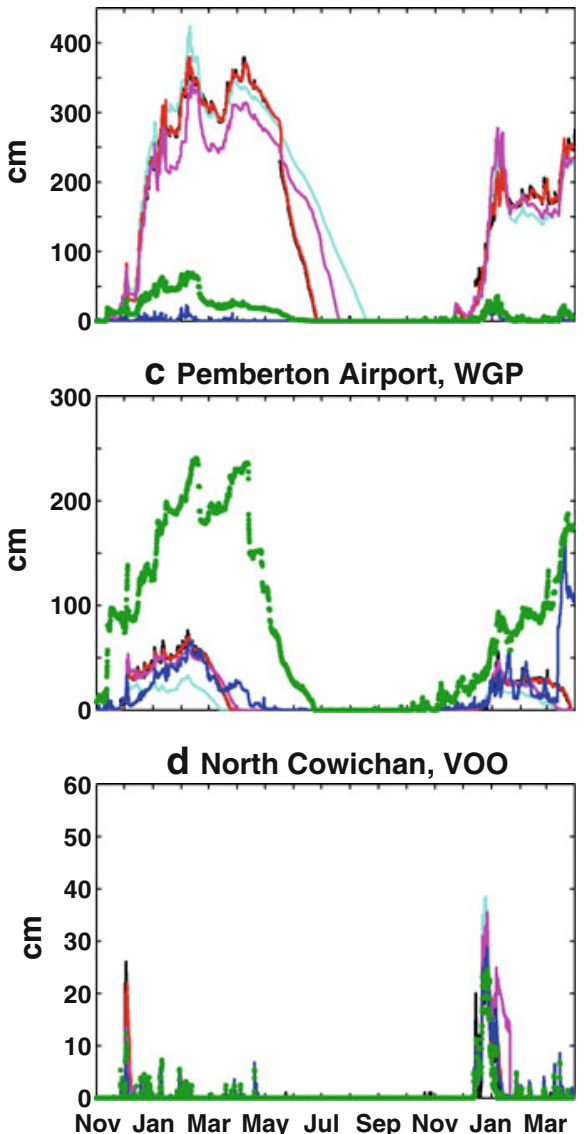

Snow Depth: Nov 2007 to Apr 2009 e Callaghan Valley, VOD

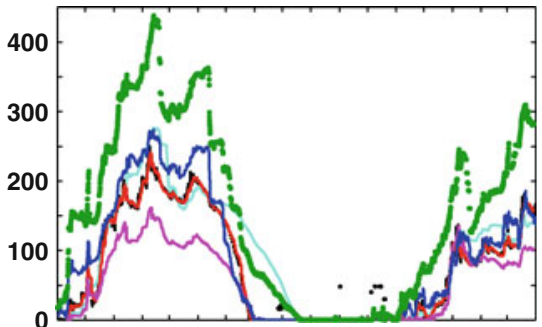

f Squamish Airport, wSK
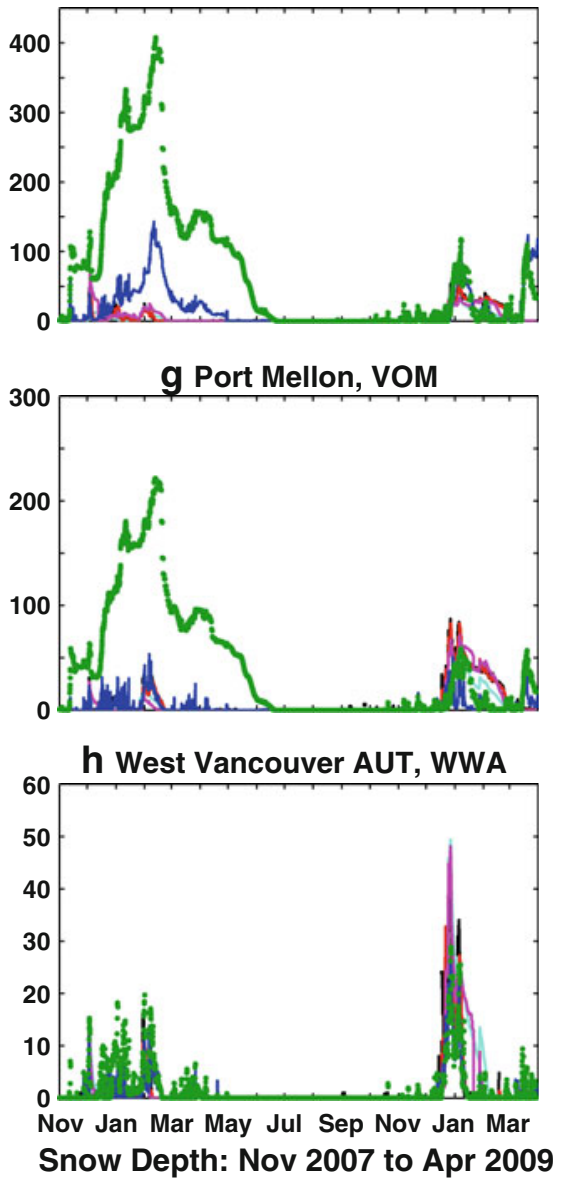

Figure 6

Snow depth forecasts at 8 stations. Black line is observed snow depth (cm). GEM-Surf three experiments are in cyan (open loop, Sect. 2.2), magenta (optimized initialization, Sect. 2.3), and red (optimized initialization and data assimilation, Sect. 2.3). GEM-15km and GEM-33km forecasts are shown in in blue and green, respectively

This extension of the snow season and the larger volumes of water stored in the overforecast snow pack compared to those in GEM-15km likely result in more humid soils which in summer result in greater dew point temperatures.
In the most basic application of GEM-Surf, snow depth is simply the result of a prognostic evolution based on relatively simple parameterizations. At low lying stations, snow depth is well forecast. At alpine stations however, the melting is initiated later than 
observed and occurs over a longer period (Fig. 6, cyan lines). It is interesting to note that snow melting is more rapid in the operational GEM- $15 \mathrm{~km}$ model despite relying on the same surface snow scheme (resolution excluded). The difference is due to the operational snow depth analysis fields in which observed temperatures above $2{ }^{\circ} \mathrm{C}$ trigger a rapid melt (BRASNETT, 1999). Since the analyses are updated daily, the snow pack decays rapidly when threshold temperatures are exceeded. This forced melting of the snow pack is not part of GEM-Surf. It is only applied to the initial conditions of the operational models.

For the first GEM-Surf experiment, the initial conditions are taken from the continuous cycle such that snow depth changes result from ISBA's simulation only. GEM-Surf cold temperature bias in both fall and winter (Fig. 4a, f) thus accumulates (in the analysis) and leads to overpredicted snow packs.

In the second GEM-Surf experiment, screen level observations for temperature and humidity were used to replace forecast fields for the initialization (the equivalent of the analysis) of surface fields. The cold fall and winter temperature bias (Fig. 4a, f) is removed from the analysis fields. This bias removal does not extend into the forecast period such that it does not affect fall and winter temperatures much, however, it results in a better timing of the melt season and melting rate (Fig. 6, magenta lines) confirming that the cold temperature bias is largely responsible for the extended snow season of the open loop forecast (Fig. 6, cyan lines).

In the last GEM-Surf experiment, snow depth data assimilation helps optimize the initial conditions and the long term evolution of the snow pack, never allowing it to depart far from the observation field (Sect. 2.4). As expected, this experiment shows the best evolution of the forecast and tracks snow depth changes well throughout all seasons (Fig. 6, red lines). It is this earlier removal of the snow cover that impacts the summer temperatures. With snow gone earlier, the net radiation is changed and GEM-Surf is able to thaw the ground and retain heat sooner leading to improved summer predictions. Another interesting aspect of the correction to the snow cover are changes in soil moisture. BERNIER and BÉLAIR (2012) showed that using GEM-Surf over an area without complex orography to provide high resolution analyses $(2.5 \mathrm{~km}$ grid spacing) to a high resolution atmospheric model (also $2.5 \mathrm{~km}$ grid spacing) leads to improved temperature and dew point forecast profiles in the first several hundred meters of the planetary boundary layer. They identified the soil moisture changes that resulted in improved snow cover analyses as the reason for the better summer forecasts.

\section{Discussion and Conclusions}

In this study, the near surface and land surface forecast and assimilation system developed for the Vancouver 2010 Olympic and Paralympic Games was presented. The system has numerous advantages over conventional forecast systems where the surface is part of a 3D atmospheric forecast model. One interesting aspect of the system is that it can easily be relocated to sites of specific importance such as airports, agricultural fields, sites of national and international events (e.g., G8 or G20 summit, Canada Games, Pan American Games, etc.) to refine forecasts according to local characteristics (e.g., vegetation types, land use, land cover, and soil type). Since the spatial scale of any one of GEM-Surf point is not limited by that of its neighboring points, the resolution can be point specific and set to reflect the complexity of local conditions (e.g., higher resolution for areas with rapidly changing orography or, numerous crop types over a small domain, and lower resolutions for large areas with single crop type).

Results presented in this study reveal that GEMSurf improves on both regional GEM- $15 \mathrm{~km}$ and global GEM-33km operational forecast models for prediction of air temperature, dew point temperature, and snow conditions. The resolution of both operational models is simply too coarse to resolve the rapidly changing orography at the boundary between the low lying coastal lands and the Rockies. The improvement gained by increasing the local resolution and thus better resolving the surface was further increased when combined with appropriate use of air temperature, dew point temperature, and snow depth observations.

In general, GEM-Surf snow depth forecasts were more representative of local conditions especially for stations such as Cypress Bowl that lie close to the 
low-lying coastal and alpine boundary. Errors in forecast peak snow pack depth for alpine stations were reduced by as much as several meters at some stations (Fig. 6). The timing of the melt season was also greatly improved in the experiment with observations driven hindcast.

Mean bias and STDE for temperature are largest in spring and summer when vegetation and soil types both contribute to increased local spatial and temporal variability in near surface air temperatures. It is expected that temperature biases could be further reduced by the addition of soil moisture data assimilation during the warm season (when the ground is not frozen). Temperature forecast improvements are smaller under fall and winter conditions although noticeable gains have been observed over a few mornings when forecast minima are no longer several degrees below the observed values. A few such gains, taken over an entire season, have little effect on the overall bias correction since their individual gain is mostly averaged out. However, taken individually, they represent a considerable improvement in forecast skill.

Dew point temperature bias was improved in GEM-Surf for all seasons. One aspect of this improvement is related to the diurnal cycle of the bias which was greatly reduced. The STDE on the other hand were most improved over the first $48 \mathrm{~h}$ of forecast time when they were often reduced by as much as $1{ }^{\circ} \mathrm{C}$. Changes in the dew point temperature forecast skill are linked to surface evaporation processes, which in winter mainly depend on surface (snow) temperature and turbulent exchange coefficients, and in summer on soil moisture. It is expected that improving the data assimilation scheme to include, for example, the assimilation of soil moisture, could further improve the forecast skill. A more advanced surface scheme, the Canadian land Data Assimilation System (CaLDAS) is currently being tested.

The system discussed here does not require training. Except for 2 months of spin-up time, it can be used to produce site specific forecasts anywhere, regardless of the availability of observations. This represents a considerable advantage over the updateable output statistics systems used at the Meteorological Service of Canada (Wilson and Vallée, 2003). The UMOS system typically requires a much longer training with observations before it can optimally be used to correct forecast fields for specific locations (e.g., GEM-15km fields) and cannot be used for a location with no observation records unlike the simple open loop form of the GEM-Surf system. The open loop, the simplest of the three configuration presented in this article, is computationally cheap in comparison to an atmospheric model, and only requires high resolution knowledge of local conditions such as orography, soil texture, and vegetation types to be used for refining lower resolution forecasts. Since such databases are available for Canada, the open loop could be used to refine operational forecasts over large regions of this country. A high resolution system (order $250 \mathrm{~m}$ ) spanning all of densely populated areas of Canada is currently in the final testing stages and should soon be used to refine operational forecasts.

Overall, the computational decoupling of the surface system from the atmospheric models allows both to run at their own time steps and relevant resolutions. The results presented here confirm considerable improvements of the near surface and land surface forecast fields. Given the improvements presented in this paper, the investigation of two-way interaction between a high resolution surface and low resolution atmospheric models in the form of fluxes from below and forcing from above is now being planned.

Work is also currently underway to improve the representation of snow processes in the ISBA land surface scheme, which will potentially have a positive impact on the modeling of snow surface temperature. The inclusion of shadow effects to account for mountain orientation is investigated. Finally, the development of a surface layer version of GEM-Surf, which may allow the improvement of low-level winds (not discussed in the present article) is also examined.

\section{Acknowledgments}

The authors wish to thank Alexandre Leroux for the acquisition of high resolution geophysical fields and Maria Abrahamowicz for her contribution to the quality control of the Olympic Network observation 
records. Special thanks are extended to Jocelyn Mailhot for the internal review of this document. Finally, thanks to the reviewers whose comments were very valuable.

Open Access This article is distributed under the terms of the Creative Commons Attribution License which permits any use, distribution, and reproduction in any medium, provided the original author(s) and the source are credited.

\section{REFERENCES}

Bélair, S., R. Brown, J. Mailhot, and B. Bilodeau, 2003a: Operational implementation of the ISBA land surface scheme in the Canadian regional weather forecast model. Part II: Cold season results. J. Hydrometeor., 4, 371-386.

Bélair, S., L.-P. Crevier, J. Mailhot, B. Bilodeau, and Y. Delage, 2003b: Operational implementation of the ISBA land surface scheme in the Canadian regional weather forecast model. Part I: Warm season results. it J. Hydrometeor., 4, 352-370.

Bélair, S., M. Roch, A.-M. Leduc, P. Vaillancourt, S. Laroche, and J. МалLнот, 2009: Medium-range quantitative precipitation forecasts from Canada's new 33-km deterministic global operational system. Wea. Forecasting, 24 (3), 690-708.

Bernier, N.B., S. Bélair, B. Bilodeau, and L. Tong. 2011: Near Surface and Land Surface Forecast for the Vancouver 2010 Olympic and Paralympic Games. J. Hydrometeor., doi: 10.1175/2011JHM1250.1, 12(4), 508-530.

BERnier, N.B., and S. BÉLAIR. 2012: High Horizontal and Vertical Resolution Limited Area Model: Near Surface and Wind Energy Forecast Applications. J. Appl. Meteor. Climatol., doi: 10.1175/JAMC-D-11-0197.1.

BRASNETT, B., 1999: A global analysis of snow depth for numerical weather prediction. J. Appl. Meteor., 38, 726-740.

Brun, E., E. Martin, V. Simon, C. Gendre, and C. Coleou. 1989: An energy and mass model of snow cover suitable for operational avalanche forecasting. J. Glacio.. 35, 333-342.

Douville, H., J.-F. Royer, and J.-F. Mahfouf, 1995: A new snow parameterization for the Météo-France climate model. Part I: Validation in stand-alone experiments. Clim. Dynam., 12, 21-35.

Gultepe, I., G.A. Isaac, P. Joe, P. Kucera, J. Theriault, T. Fiziko, and K. UNGAR, 2012: RND mountain top research site: measurements and uncertainties. Pure and Appl. Geophys., (this issue).
Isaac G.A., P.I Joe, J. Mailhot, M. Bailey, S. Bélair, F.S. Boudala, M. Brugman, E. Campos, R.L. Carpenter Jr, R.W. Crawforsd, S.G. Cober, B. Denis, C. Doyle, H.D. Reeves, I. Gultepe, T. Haiden, I. Heckman, L.X. Huang, J.A. Milbrandt, R. Mo, R.M. Rasmussen, T. Smith, R.E. Steward, D. Wang, and L.J. WILSON, 2012. Science of Nowcasting Olympic Weather for Vancouver 2010 (SNOW-V10): A World Weather Research Programme Project. Pure and Appl. Geophys., (this issue).

Joe P., B. Scott, C. Doyle, G.A. Isaac, I. Gultepe, D. Forsyth, S.G. Cober, E. Campos, I. Heckman, N. Donaldson, D. Hudak, R. Rasmussen, R.E. Steward, J.M Thériault, H. Carmichael, M. BAILey, and F.S. Boudala, 2012. The monitoring Network of the Vancouver 2010 Olympics. Pure and Appl. Geophys., (this issue). JORDAN, R.E., 1991:A one-dimensional temperature model for a snow cover. Technical documentation for SNTHERM.89, Special Rep. 91-16, US Army Cold Reg. Res. and Eng. Lab., Hanover, N. H., United States of America.

Mailhot, J., S. Bélair, L. Lefaivre, B. Bilodeau, M. Desgagné, C. Girard, A. Glazer, A.-M. Leduc, A. Methot, A. Patoine, A. Plante, A. Rahill, T. Robinson, D. Talbot, A. Tremblay, P. VAillancourt, A. ZADRA, and A. QADDOURI, 2006: The 15-km version of the Canadian regional forecast system. Atmos.-Ocean, 44 (2), 133-149.

Mailhot J., S. Bélair, M. Charron, C. Doyle, P. Joe, M. Abrahamowicz, N.B. Bernier, B. Denis, A. Erfani, R. Frenette, A. Giguère, G.A. Isaac, N. McLennan, R. McTaggart-Cowan, J. Milbrandt, and L. Tong. 2010: Environment Canada's Experimental Numerical Weather Prediction Systems for the Vancouver 2010 Winter Olympic and Paralympic Games, Bull. Amer. Meteor. Soc., doi:10.1175/2010BAMS2913.1, 91,1073-1085

Mailhot, J., J. Milbrandt, A. Giguère, R. McTaggart-Cowan, A. Erfani, B. Denis, A. Glazer, and M. Vallée, 2012. An experimental 1-km resolution forecast model during the 2010 Vancouver Winter Olympic and Paralympic Games. Pure and Appl. Geophys., (this issue).

Nollhan, J. and S. Planton, 1989: A simple parameterization of land surface processes for meteorological models. Mon. Wea. Rev., 117, 536-549.

SLATER, A. G., et al., 2001: The representation of snow in land surface schemes: Results from PHILPS 2(d). J. Hydrometeor., $7-25$.

Wilson, L. J. and M. Vallée, 2003: The Canadian Updateable Model Output Statistics (UMOS) system: Validation against perfect prog. Wea. Forecasting, 18, 288-302.

Zadra, A., M. Roch, S. Laroche, and M. Charron, 2003: The subgrid-scale orographic blocking parametrization of the GEM model. Atmos.-Ocean, 41 (2), 155-170. 